\title{
A Review on EBG Based Power Dividers
}

\author{
Pranavi Mhatre, Jyoti. M. Varavdekar \\ (EXTC Department, K.J. Somaiya CoE, University of Mumbai, India)
}

\begin{abstract}
In the recent years, there has been considerable activity in the area miniaturization of microwave devices. Miniaturization offers the benefits of low material costs, high production output and potentially faster operation. It can be achieved through various techniques such as 'Defected Ground Structure' (DGS), 'Electromagnetic Bandgap' (EBG), etc. This paper is a comprehensive review of EBG Based Power Dividers. The objective of the paper is to provide the readers with a broad perspective of the principle behind miniaturization of power dividers using EBG and the different EBG structures used for miniaturization of power dividers.
\end{abstract}

Keywords -Electromagnetic Bandgap (EBG), Harmonic Suppression, Hybrid Ring, Miniaturization, Power Divider, Slow Wave Structure (SWS)

\section{INTRODUCTION}

Power Dividers are one of the key components in microwave circuits, and are widely used in balanced amplifiers, the feeding networks of an antenna array, etc [1]. Power Dividers couple a defined amount of the electromagnetic power in a transmission line to a port enabling the signal to be used in another circuit. An essential feature of directional couplers is that they only couple power flowing in one direction. Techniques like EBG aid in reducing the size of the power divider. Miniaturization is an important factor in designing overall compact microwave systems. In this paper, the concept of EBG is discussed. The next section presents the conventional power divider and different EBG structures used in power dividers are discussed in the following sections.

\section{ELECTROMAGNETIC BANDGAP: THE CONCEPT}

Electromagnetic Bandgap (EBG) structures are periodic structures with ability to control the propagation of electromagnetic waves [2]. The EBG structures introduce a defect in the conventionally continuous microwave structure. Such defects increase the effective inductance and capacitance. The current now encounters more impedance than it would in absence of the defect and hence its velocity decreases. Hence, the structure is also termed as SWS (Slow Wave Structure).

$$
\begin{aligned}
& V p=\frac{1}{\sqrt{V_{C}}} \\
& \lambda_{g}=\frac{V_{g}}{f} \\
& \varepsilon=\left(\frac{A}{\lambda_{g}}\right)^{2} \\
& Z_{0}=\sqrt{\frac{I}{C}}
\end{aligned}
$$

In (1), $\mathrm{L}$ represents the inductance and $\mathrm{C}$ represents capacitance. As $\mathrm{L}$ and $\mathrm{C}$ increase, the phase velocity $\mathrm{Vp}$ decreases; thus demonstrating the 'Slow Wave Effect'. As Vp decreases, the guide wavelength $\lambda \mathrm{g}$ decreases too as shown in (2). As $\lambda \mathrm{g}$ decreases, the effective permittivity $\varepsilon$ increases as in (3). Here, $\lambda$ is the free space wavelength. This increases in $\varepsilon$ leads to miniaturization of the device. The idea of EBG structure formulation is to increase the $\mathrm{L}$ and $\mathrm{C}$ such that the phase velocity $\mathrm{Vp}$ is lowered while maintaining the ratio $\mathrm{L} / \mathrm{C}$ so that the characteristic impedance Zo is not disturbed as in (4).

\section{EBG STRUCTURES FOR POWER DIVIDERS}

In [3], an EBG based Wilkinson Power Divider using capacitive loading with 50\% size reduction as compared to conventional power divider and is presented. A conventional Wilkinson power divider is composed of two quarter-wavelength transmission-line sections at the designed frequency, which results in a large occupied area. Fig. 1 shows the conventional power divider and the EBG based power divider proposed in the paper. The transmission line section at the designed frequency of a conventional divider is substituted by one cell of EBG structure. 

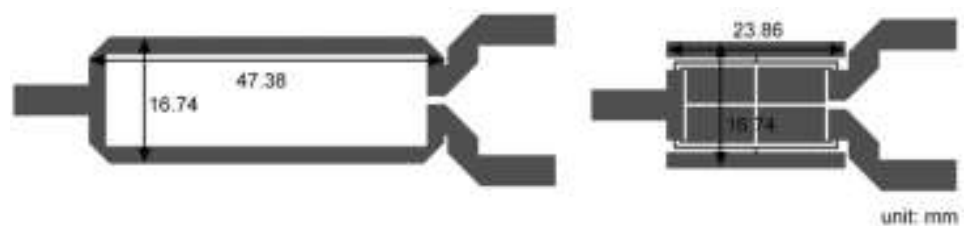

Fig. 1. Size comparison of the proposed and conventional dividers [3]

In [4], the EBG technique is used for suppression of harmonics in the output of the power divider. A size reduction of $30 \%$ has been achieved for the quarter wave line. $32.5 \mathrm{~dB}$ suppression is achieved for the third harmonic and $12 \mathrm{~dB}$ suppression is achieved for the fifth harmonic in the design proposed. In this EBG structure, perforations are made on the transmission line. The proposed power divider consists of two microstrip EBG cells and four microstrip lines as shown in Fig. 2(a).

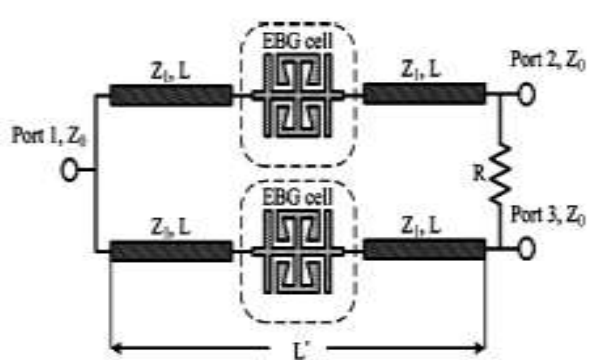

(a)

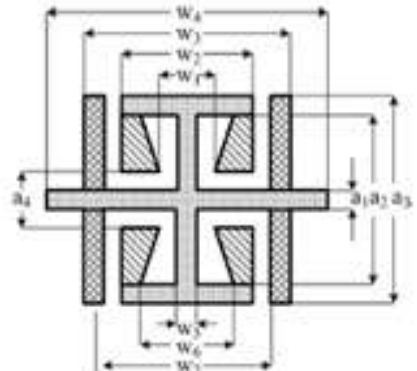

(b)

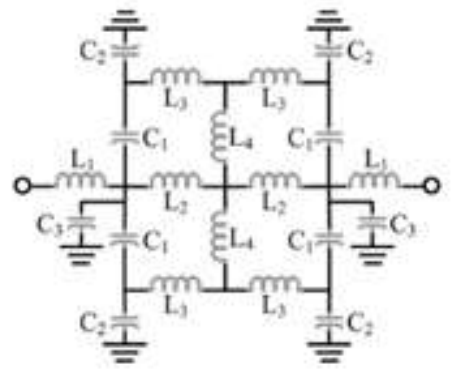

(c)

Fig. 2. (a) Schematic diagram of the proposed power divider for harmonic suppression (b) Proposed microstrip EBG cell. (c) Lossless L-C equivalent circuit [4]

In Fig. 2 (b), the structure of the EBG cell is shown. This EBG cell can provide two resonances to suppress the unwanted harmonics and reduce the length of a quarter-wave line simultaneously. By adjusting the narrow connecting lines and the size of the etched pattern, different slow-wave effects at different frequencies can be obtained to suppress the unwanted nth harmonics. in Fig. 3.

In [5], a dual band Wilkinson Power Divider using Mushroom like EBG structure is proposed as shown

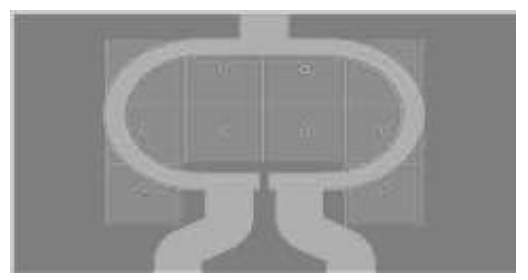

Fig. 3. Geometry of the proposed dual-band power divider over the EBG structure [5]
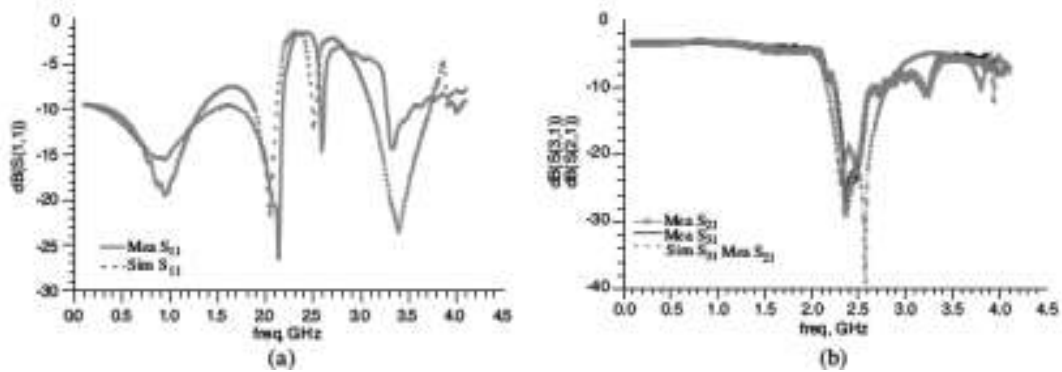

Fig 4. Comparison of simulated and measured magnitudes of the proposed dual-band power divider (a) $S 11$ (b) $S 21$ and $S 31$ [5]

The power divider operates at $1 \mathrm{GHz}$ and $2 \mathrm{GHz}$. A mushroom-like periodic metallic pattern is implemented for EBG structure. Each unit cell comprises two elements; a patch and a via. The EBG structure generates a band-gap in the specific frequency range and no signal is received at the output ports in this range. The via is connected between the patch and ground plane. The size of patch gap between patches and the radius of via affect the band-gap frequency.

In [6], a 6:1 Unequal Wilkinson Power Divider with EBG CPW has been proposed. For a divider with highly unequal power ratios, a microstrip line having very high characteristic impedance is required. For 6:1 
power divider, a microstrip line of $270 \Omega$ is needed. Such traces are very narrow and difficult to fabricate. The proposed EBG CPW not only has a wider strip but also achieves high impedance. The ground planes are connected to each other using via holes, thus avoiding the undesired waveguide modes generated.

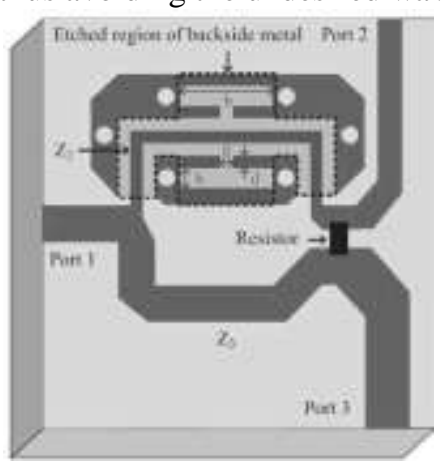

Fig 5. A schematic diagram of the proposed 6:1 unequal Wilkinson power divider [6]

In [7], an In-Phase Hybrid Ring Power Divider operating at $4 \mathrm{GHz}$ has been designed using the two types of EBG structures. The paper also presents detailed analysis of the EBG structure by approximating its electrical model. The EBG cell and its electrical model are shown in Fig. 6.

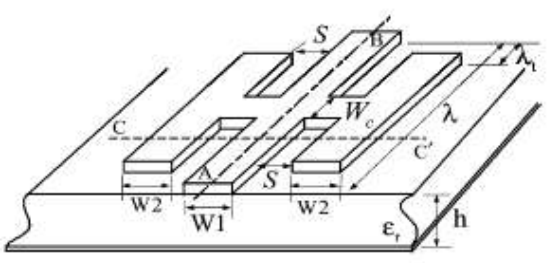

(a)

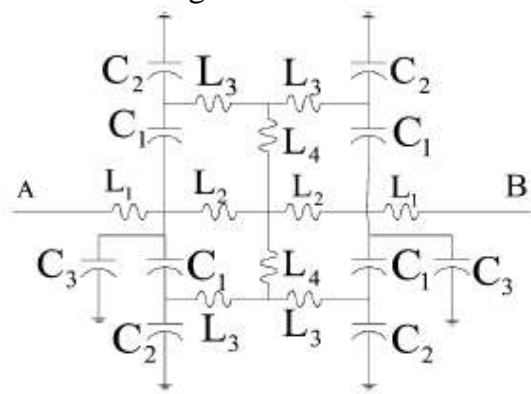

(b)

Fig 6. (a) Typical EBG unit cell. (b) Lossless equivalent circuit [7]

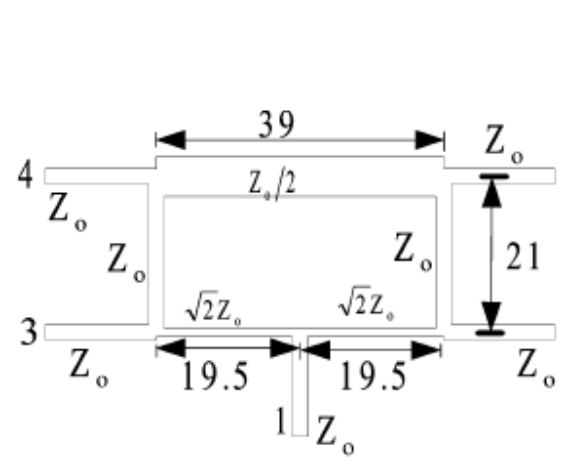

(a)

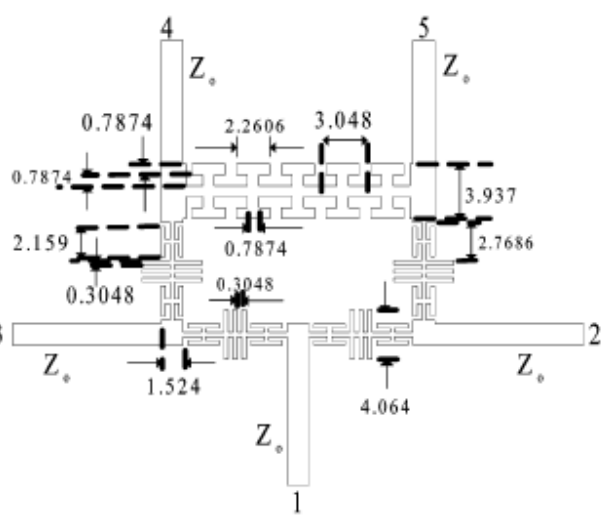

(b)

Fig. 7. (a) Conventional in-phase hybrid-ring equal power divider (b) Proposed structure [7]

One type of EBG unit cells are etched along the ports 2 and 3, and another type of EBG cells along ports 4 and 5. The use of these two types of EBG cells provides better matching at port 1 . The vertical oriented EBG unit cell along ports 2 and 3 serve as the required stub matching for port 1 . Since ports 4 and 5 are the termination ports for matching ports 2 and 3 , the length from ports 3 to 4 or ports 2 to 5 is already sufficient to provide the required matching. Hence, one type of EBG unit cells are etched along ports 4 and 5. 


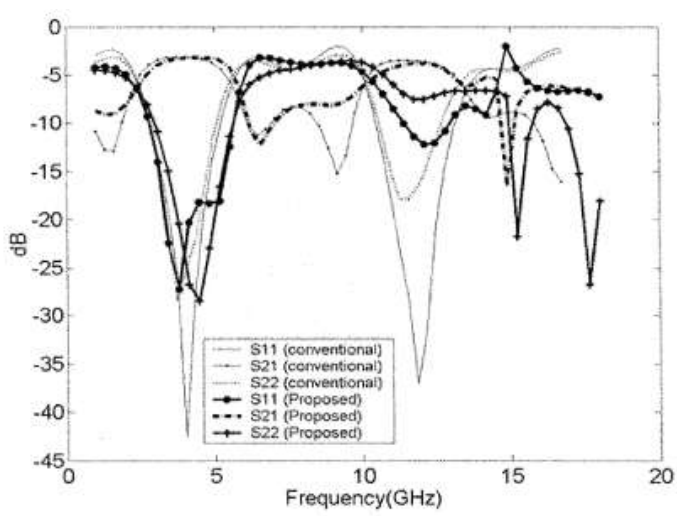

Fig. 8. Comparison of results of Conventional Design and Proposed Design

The proposed EBG based design offers larger bandwidth over conventional design as seen in Fig. 8. It also suppresses harmonics in the range of 10-14 GHz. Since the length of the proposed design is shorter than the conventional design, lower insertion loss is seen in the former.

\section{CONCLUSION}

Different EBG structures for power divider miniaturization were presented in this review. In comparison to the conventional design, power divider designed using EBG structures are more compact, have a larger bandwidth and offer good harmonic suppression. The EBG structures can be electrically modeled and hence can be reproduced for operating at other frequencies than the one designed for by varying the design structure parameters.

\section{REFERENCES}

[1] Pozar, D. M., Microwave Engineering, 3rd Edition, Wiley, New York, 2005.

[2] Dusan Nesie, , Frequency A Brief Review of Microwave Photonic Bandgap (PBG) Structures, Microwave Review, pp July 2001

[3] Jun He1, Ding Chen, and Bing-Zhong Wang, Miniaturized Microstrip Wilkinson Power Divider with EBG Structure, Microwave and Millimeter Wave Technology (ICMMT), 2012 International Conference on , vol.4, no., pp.1-3, 5-8 May 2012

[4] Chih-Ming Lin; Hsuan-Hung Su; Jui-Chieh Chiu; Yeong-Her Wang; , Wilkinson Power Divider Using Microstrip EBG Cells for the Suppression of Harmonics, Microwave and Wireless Components Letters, IEEE, vol.17, no.10, pp.700-702, Oct. 2007

[5] Hsin-Hao Chen and Yi-Hsin Pang, A Dual-band Wilkinson Power Divider Utilizing EBG Structure, PIERS Proceedings, Marrakesh, MOROCCO, March 20-23, 2011, pp 1018-1020

[6] C.-P. Chang, C.-C. Su, S.-H. Hung, and Y.-H. Wang, A 6:1 Unequal Wilkinson Power Divider With EBG CPW, Progress In Electromagnetics Research Letters, Vol. 8, 151-159, 2009

[7] Ban-Leong Ooi,, Compact EBG In-Phase Hybrid Ring Equal Power Divider, IEEE TRANSACTIONS ON MICROWAVE THEORYAND TECHNIQUES, VOL. 53, NO. 7, JULY 2005, pp 2329-2334 